\title{
Female-limited variability in mimicry in the swallowtail butterfly Papilio polyxenes Fabr.
}

\author{
JENNIFER HERRELL \& WADE HAZEL* \\ Department of Biological Sciences, DePauw University, Greencastle, IN 46135, U.S.A.
}

\begin{abstract}
Variation in dorsal and ventral aspects of mimetic wing patterns was measured in the sexually dimorphic swallowtail butterfly Papilio polyxenes, a Batesian mimic of Battus philenor. The pattern on the dorsal surface of the wings makes females better mimics than males and was more variable in females. The pattern on the ventral surface of the hind wings, which provides a similar degree of mimicry in both sexes, was equally variable in both sexes. Full-sib ANOvA indicated that variability in dorsal and ventral patterns was similarily heritable in both sexes, suggesting that the increased variability in females is not simply the result of suppressed expression of genetic variation in males. The results are consistent with the hypothesis that variation in two sets of modifier loci, only one of which is expressed in males, influences the dorsal pattern, resulting in greater variability in females. The nonmimetic dorsal pattern in males is probably maintained as a consequence of sexual selection arising from male-male competition in territorial encounters. The generality of the reduced male variability in the Lepidoptera is discussed.
\end{abstract}

Keywords: evolution, female-limited variation, mimicry, Papilio polyxenes.

\section{Introduction}

A number of authors have suggested that the suppression of variation is a general characteristic of male Lepidoptera (Wallace, 1865; Fisher \& Ford, 1928). Morphological studies have found reduced male variability in a wide range of traits in butterflies including coloration (Fisher \& Ford, 1928), spot size and frequency (Pearse \& Murray, 1982) and wing and tail length in swallowtail butterflies (Clarke \& Sheppard, 1962a). Polymorphisms in Batesian mimicry, where whole suites of traits conferring mimicry of different model species vary only in females, are classic examples of the phenomenon (Turner, 1978).

Genetic mechanisms for reduced male variability are reviewed in Pearse \& Murray (1982). All involve regulatory interactions. The simplest model, proposed by Johnson \& Turner (1979), supposes the suppression of autosomal loci by two doses of an X-linked regulatory locus (males are the homogametic sex in the Lepidoptera). Complementary evolutionary processes that might generate reduced variability in males include female choice, male-male competition, and habitat separation (Brower, 1963; Turner, 1978; Pearse and Murray, 1982; Silberglied, 1984).

*Correspondence.
In a recent study, Hazel (1990) examined wing pattern variation in the swallowtail butterfly Papilio polyxenes, a Batesian mimic of the pipevine swallowtail, Battus philenor (Brower, 1958; Jeffords et al., 1979). Male and female Papilio polyxenes resemble $B$. philenor in having largely black wings and a row of orange spots on the ventral hindwings (Fig. 1), which makes them effective mimics when the ventral surfaces are visible (i.e. when the wings are folded) (Codella \& Lederhouse, 1989). On the dorsal surfaces of the wings two bands of yellow spots (proximal and distal) run anterior to posterior on the forewings and hindwings. The spots of the proximal band in males are conspicuous, and all eight spots are usually present on each wing. In females, however, the proximal band spots are less conspicuous and some spots are often absent (Hazel, 1990). Because B. philenor lacks spots on the dorsal surfaces of its wings, female $P$. polyxenes are, overall, better mimics than are males (Codella \& Lederhouse, 1989). The number of spots comprising the proximal band is not only fewer in females, but it is also more variable than in males. In addition, variation in spot number in females, and therefore degree of mimicry, is heritable, suggesting that genetic variation is being suppressed in males (Hazel, 1990).

The black colour of $P$. polyxenes wings is determined by a dominant autosomal allele $(B)$ (Clarke \& Sheppard, 1955). In related species (e.g. P. zelicaon, 

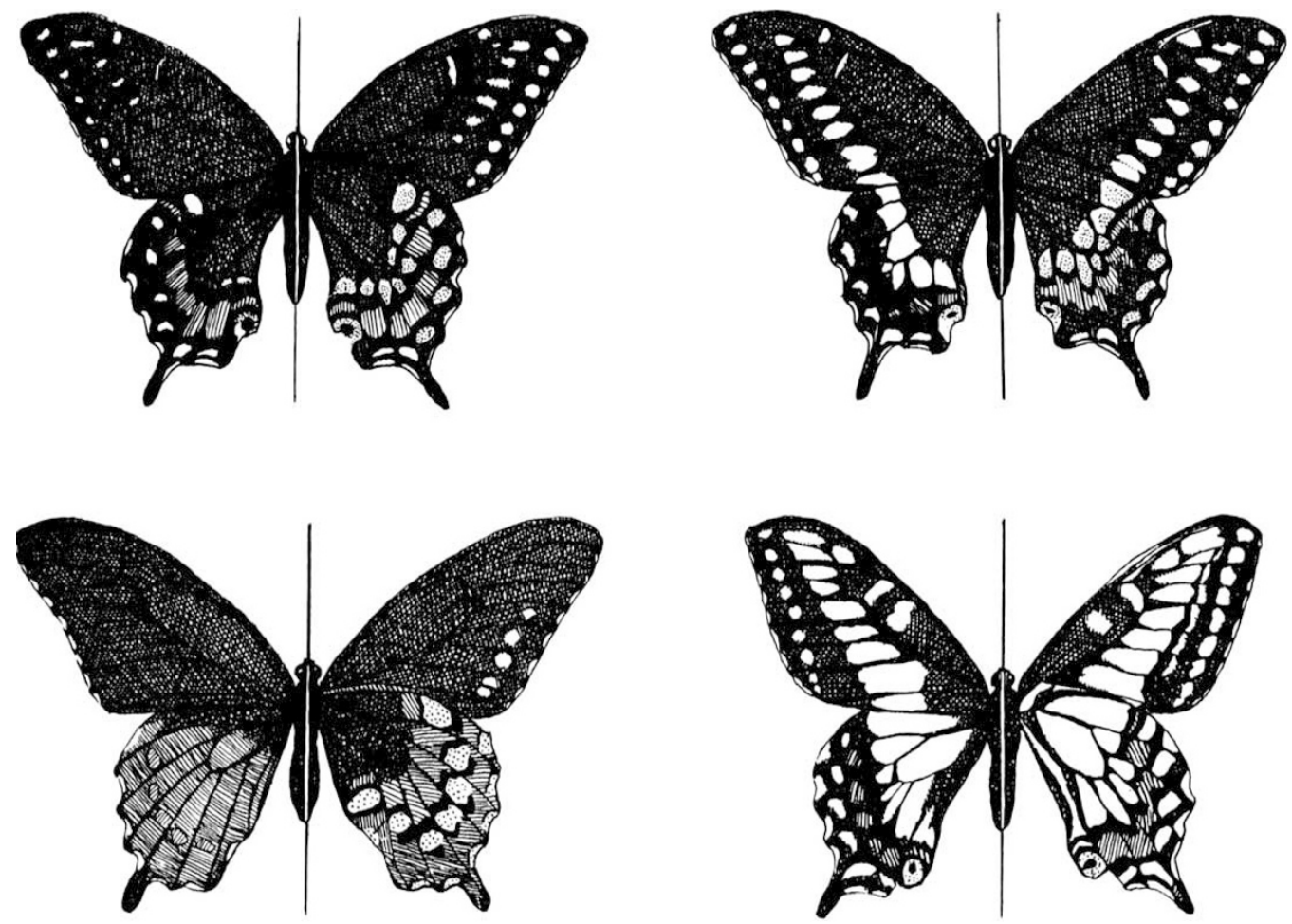

Fig. 1 Wing patterns of female and male Papilio polyxenes (upper left and right, respectively), Battus philenor (lower left), and $P$. zelicaon (lower right). For each butterfly, dorsal and ventral wing surfaces are on the left and right, respectively. Crosshatched $=$ black, striped $=$ blue, stippled $=$ orange, and open areas = yellow. Note the prominent proximate yellow band in $P$. zelicaon and male P. polyxenes.

Fig. 1) homozygous for the recessive allele $(b)$ the amount of black is greatly reduced and the width of the proximal band of yellow spots is increased. Hazel (1990) hypothesized that increased expression of the $B$ allele and its modifiers in female $P$. polyxenes could reduce the width of the spot band to the point where some spots are eliminated. The hypothesis is consistent with developmental models of wing pattern formation in butterflies (Nijhout, 1991), and would explain why females had lower and more variable numbers of spots. In males, reduced expression of the $B$ allele and its modifiers would rarely result in the elimination of spots and, therefore, spot number would vary little. If this hypothesis is correct, then the lack of heritable variation in mimicry in males (Hazel, 1990) is simply a consequence of measuring variation in mimicry in terms of spot number, and the size of the yellow spot band should show similar levels of variability and heritability in males and females. If, however, the suppression of genetic variability is a general feature of male butterflies, then the sizes of the yellow spot band on the dorsal wing surfaces and the orange spot band on the ventral surface of the hindwings should be more variable and more heritable in females.

\section{Materials and methods}

The butterflies used in this study were the offspring of wild-caught females taken from a single population in Putnam Co., Indiana, USA, in 1984. Larvae were reared in plastic rearing dishes on a $12 \mathrm{~L}: 12 \mathrm{D}$ photoperiod at room temperature (approx. $30^{\circ} \mathrm{C}$ ), and were supplied with fresh foodplant (Daucus carota) daily. Only butterflies eclosing from pupae that failed to enter diapause (eclosion within six weeks of pupation) were used in this study. After eclosion, wings were removed and laminated to index cards for video-taping and measurement.

Wing patterns were digitized from video-tape and measurements were taken using the JAVA video analysis system (Jandel Scientific, Corte Madera, CA, USA). Measurements of the same individuals were found to be highly repeatable (repeatability $=0.99,10$ individuals measured five times each). Wing size (area) and the area of each spot in the proximal yellow spot band on the dorsal surfaces of the wings were measured. Spot sizes were then summed to obtain a single measure of size of the spot band in $\mathrm{mm}^{2}$. The size of the ventral orange spot band on the hind wing was determined 
similarly, except that, for technical reasons, the area was measured in pixels. To eliminate any effects arising from differences in wing size, spot band sizes were regressed on wing size separately for each sex, and the regression equations were used to standardize spot band size to the average wing size. Coefficients of variation were used to compare the relative variabilities of spot band sizes in males and females.

Estimates of the genetic component of variation in spot band size were calculated separately for males and females using one-way ANOVA of full-sib families (19 families: five butterflies/family). The assumption that all offspring of a single wild-caught female are full-sibs is based on evidence of sperm precedence in this species (Clarke \& Sheppard, 1962b). Because the between-families component of variance, in addition to including additive and dominance components, will also include any maternal effects, our estimates of the genetic component of variation may be biased upwards (Falconer, 1981).

\section{Results}

The proximal band was much larger in males than in females, with no overlap in size distributions $(P<0.001$, two sample $t$-test $)$. However, the amount of yellow varied more in females than in males (coefficients of variation of 15 per cent \pm 1 per cent and 53 per cent \pm 4 per cent for males and females, respectively). Differences in variability are readily apparent when the amount of yellow is expressed relative to the mean (Fig. 2). The amount of orange on the ventral hind wings was greater in males $(P<0.001$, two sample $t$-test), although there was considerable overlap in the distributions (means and standard deviations were $1531 \pm 366$ pixels for females and $2056 \pm 488$ pixels

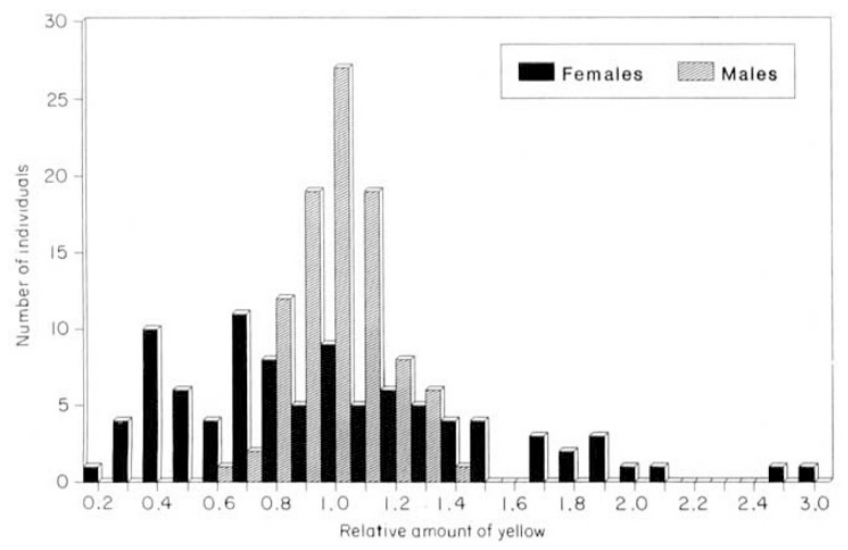

Fig. 2 Relative amount of variation in the size of the proximal yellow spot band (size of spot band/average size of spot band) on the dorsal surface of the wings of female and male Papilio polyxenes. for males), and the coefficients of variation were the same (23 per cent in each) (Fig. 3).

The size of the proximal yellow band on the dorsal surfaces of the wings and the orange band on the ventral hindwings varied significantly between families in both sexes (Tables 1 and 2). For females, 44 per cent

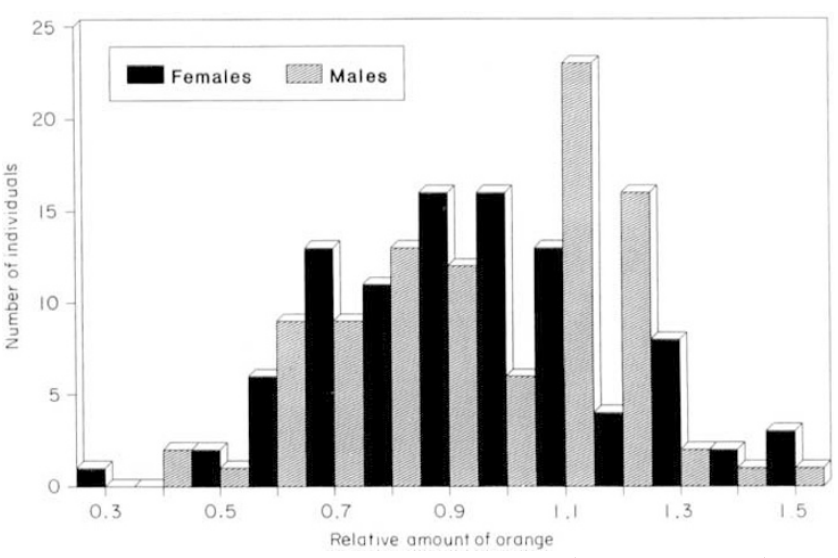

Fig. 3 Relative amount of variation in the size of the orange proximal spot band (size of spot band/average size of spot band) on the ventral surfaces of the hindwings in female and male Papilio polyxenes.

Table 1 One-way analyses of variance of size of the yellow proximal spot band on the dorsal surfaces of the wings of Papilio polyxenes females and males

\begin{tabular}{lllc}
\hline Source & d.f. & MS & $F$ \\
\hline (a) Females & & & \\
$\quad$ Between families & 18 & 222.1 & $4.98^{*}$ \\
$\quad$ Within families & 76 & 44.6 & \\
(b) Males & & & \\
$\quad$ Between families & 18 & 663 & $4.08^{*}$ \\
$\quad$ Within families & 76 & 163 & \\
$* P<0.001$. & & &
\end{tabular}

Table 2 One-way analyses of variance of size of the orange proximal spot band on the ventral surface of the hindwings of Papilio polyxenes females and males

\begin{tabular}{lccc} 
Source & d.f. & MS & $F$ \\
\hline $\begin{array}{l}\text { (a) Females } \\
\quad \text { Between families }\end{array}$ & 18 & 233292 & $2.11^{*}$ \\
$\quad$ Within families & 76 & 110606 & \\
$\begin{array}{l}\text { (b) Males } \\
\quad \text { Between families }\end{array}$ & 18 & 527513 & $3.11^{* *}$ \\
$\quad$ Within families & 76 & 169372 & \\
\hline * $P<0.05,{ }^{* *} P<0.001$. & & & \\
& & & \\
(c) The Genetical Society of Great Britain, Heredity, 75, 106-110.
\end{tabular}


and 18 per cent of the variance in amount of yellow and orange, respectively, was attributable to differences between families. For males, comparable values for the between-families components were 38 per cent and 30 per cent for amount of yellow and orange, respectively. Assuming differences between families reflect only additive effects, heritability estimates based on these data are not significantly different (amount of yellow: female $h^{2}=0.89$, male $h^{2}=0.76$; amount of orange: female $h^{2}=0.36$, male $h^{2}=0.59$ ).

\section{Discussion}

Our results provide additional evidence for the suppression of phenotypic variation in male butterflies. The size of the proximal band on the dorsal surfaces of the wings of $P$. polyxenes is not only smaller in females, but more variable than in males. These results parallel those of Hazel (1990), which showed that female $P$. polyxenes had lower and more variable numbers of spots than did males. However, the results do not support the hypothesis that the decreased number of spots in the proximal spot band and increased variability in spot number in females were caused by slight differences in the expression of the same genes in females and males (Hazel, 1990), because the prediction that variation in the size of the proximal spot band should be the same in males and females is not supported. In addition, our results, showing that variation in both sexes is similarly heritable, provide no evidence that reduced variability results from the general suppression of genetic variation underlying wing pattern in males. If this were the case, the fraction of variation attributable to differences between families would have been smaller in males than in females.

The pattern of variability in the proximal yellow band can be most easily explained by postulating genetic variation in two groups of genes that modify the phenotypic effect of the $B$ allele (Clarke and Sheppard, $1955)$, with one group active in both sexes and the other active only in females. If the combined effects of the two groups are additive, females will be more variable than males, but variation in both sexes will be heritable. This mechanism is consistent with the classic view of Fisher (1927) that mimicry evolves as a result of selection for modifers that alter the effects of genes with major effects (e.g. the $B$ gene in $P$. polyxenes). In the initial stages of the evolution of mimicry in $P$. polyxenes, modifiers of the $B$ gene that improved mimicry by decreasing the size of the proximal band in both sexes would have been favoured. Then, assuming the presence of the proximal band to be of adaptive significance only in males (see below), selection would have favoured modifiers whose expression was limited to females.

The hypothesis above is also consistent with the results of hybridizations between $P$. polyxenes and related species (Clarke \& Sheppard, 1955). For example, Clarke \& Sheppard (1955) found that in $F_{1}$ hybrids of $P$. polyxenes and $P$. machaon or $P$. zelicaon, both of which normally lack the $B$ allele, the inner margin of the proximal band of yellow spots is less well defined than in $P$. polyxenes. They suggested that selection must have favoured a 'gene-complex' in $P$. polyxenes that gives the proximal band a distinct out line. In addition, when $P$. polyxenes is hybridized with $P$. brevicauda, which is homozygous for the $B$ allele, but is not sexually dimorphic (both sexes look like $P$. polyxenes males), the $F_{1}$ progeny have reduced levels of sexual dimorphism. Clarke \& Sheppard (1955) interpreted this as reflecting a difference in 'the residual gene-complex of the two species'. Interestingly, $P$. polyxenes $\times P$. zelicaon hybrids and $P$. zelicaon backcross progeny heterozygous for the $B$ allele show the sexual dimorphism characteristic of $P$. polyxenes, indicating that modifiers of the $B$ allele are also present in $P$. zelicaon.

Three explanations have been proposed for the lack of both mimicry and variability in male butterflies: female choice (Brower, 1963; Turner, 1978), male-male competition (Pearse \& Murray, 1982; Silberglied, 1984), and habitat separation (Brower, 1963; Turner, 1978). Of these, male-male competition provides the most reasonable explanation for reduced variability and increased prominence of the yellow spot band in male $P$. polyxenes. Male and female $P$. polyxenes fly together in the open field habitats where males compete for high ranking territories (Lederhouse, 1982). The proximal yellow spot band apparently influences the outcome of this intrasexual competition (Lederhouse, 1995; Lederhouse and Scriber, in press). Experimental elimination of the proximal band in males has no effect on female choice, but does decrease the likelihood that a modified male will be able to obtain a high ranking territory. Because for males, the advantages of obtaining and holding a territory are probably greater than those of mimicry, selection has favoured the retention of the proximal yellow band.

Finally, there are two senses in which female-limited variability might be considered a general feature of the Lepidoptera. First, female-limited variability might be the general rule for the group as a whole; and second, in species in which female-limited variability occurs, many aspects of the phenotype might be affected. Pearse \& Murray (1982) have questioned the generality of female-limited variability in the Lepidoptera as a 
group. Based on their analysis of the taxonomic distribution of single-locus sex-limited variability, they concluded that sex-limited polymorphisms are rare in both moths and butterflies and are 'spectacularly clustered within particular taxa', for example, among swallowtails in the tribe Papilionini. Our results question the generality of female-limited variability in the second sense: in $P$. polyxenes, female-limited variability affects only the proximal yellow spot band on the dorsal surface of the wings, but not the proximal band of orange spots on the ventral surface of the hindwings. Therefore, female-limited variability may be less general than previously thought.

\section{Acknowledgements}

M. Frontz assisted in the data collection. Barbara Field-Timm drew the butterflies in Fig. 1. Jim Benedix, David West, Bob Lederhouse and two anonymous reviewers provided suggestions for improving the manuscript. This research was supported by a grant from the Howard Hughes Medical Institute to DePauw University.

\section{References}

BROWER, J. VAN Z. 1958. Experimental studies of mimicry in some North American butterflies. Part II. Battus philenor and Papilio troilus, $P$. polyxenes, and $P$. glaucus. Evolution, 12, 123-136.

BROWER, L. P. 1963. The evolution of sex-limited mimicry in butterflies. Proc. XVI Int. Congr. Zool., Wash., 4, 173-179.

CLARKE, C. A. AND SHEPPARD, P. M. 1955. A preliminary report on the genetics of the machaon group of swallowtail butterflies. Evolution, 12, 123-136.

ClARKE, C. A. AND SHEPPARD, P. M. 1962a. Disruptive selection and its effect on a metrical character in the butterfly Papilio dardanus. Evolution, 16, 214-226.

ClARKE, C. A. AND SHEPPARD, P. M. 1962b. Offspring from double matings in swallowtail butterflies. The Entomologist, 95, 199-203.

CODELLA, S. G. AND LEDERHOUSE, R. C. 1989. Intersexual comparison of mimetic protection in the black swallowtail butterfly, Papilio polyxenes: experiments with captive blue jay predators. Evolution, 43, 410-420.
FALCONER, D. S. 1981. Introduction to Quantitative Genetics, 2nd edn. Longman, London and New York.

FISHER, R. A. 1927. On some objections to mimicry theory; statistical and genetic. Trans. R. Ent. Soc. Lond., 75, 269-278.

FISHER, R. A. AND FORD, E. B. 1928. The variability of species in the Lepidoptera with reference to abundance and sex. Trans. R. Ent. Soc. Lond., 76, 367-379.

HAZEL, W. N. 1990. Sex-limited variability and mimicry in the swallowtail butterfly Papilio polyxenes Fabr. Heredity, 65 , 109-114.

JEFFORDS. M. R., STERNBURG, J. G. AND WALDBAUER, G. P. 1979. Batesian mimicry: field demonstration of the survival value of pipevine swallowtail and monarch color patterns. Evolution, 33, 275-286.

JOHNSON, M. S. AND TURNER, J. R. G. 1979. Absence of dosage compensation for a sex-linked enzyme in butterflies (Helicoinus). Heredity, 43, 71-77.

LEDERHOUSE, R. C. 1982. Territorial defense and lek behavior of the black swallowtail butterfly, Papilio polyxenes. Behav. Ecol. Sociobiol., 10, 109-118.

LEDERHOUSE, R. C. 1995. Comparative mating behavior and sexual selection in North American swallowtail butterflies. In: Scriber, J. M., Tsubaki, Y. and Lederhouse, R. C. (eds) Swallowtail Butterflies: Their Ecology \& Evolutionary Biology, pp. 117-131. Scientific Publishers, Inc., Gainesville, FL.

LEDERHOUSE, R. C. AND SCRIBER, J. M. 1995. Intrasexual selection constrains the evolution of the dorsal color pattern of male black swallowtail butterflies, Papilio polyxenes. Evolution.

NIJHOUT, F. H. 1991. The Development and Evolution of Butterfly Wing Patterns. Smithsonian Institution Press, Washington and London.

PEARSE, F. K. AND MURRAY, N. D. 1982. Sex and variability in the common brown butterfly Heteronympha merope merope (Lepidoptera: Satyrinae). Evolution, 36, 1251-1264.

SILBERGLIED, R. E. 1984. Visual communication and sexual selection among butterflies. In: Vane-Wright, R. I. and Ackery, P. R. (eds) The Biology of Butterflies, Symposium of the Royal Entomological Society of London, Number 11, pp. 207-223. Academic Press, London.

TURNER, J. R. G. 1978. Why male butterflies are non-mimetic: natural selection, sexual selection, group selection, modification and sieving. Biol. J. Linn. Soc., 10, 385-432.

WALlACE, A. R. 1865 . On the phenomena of variation and geographical distribution as illustrated by the Papilionidae of the Malay region. Trans. Linn. Soc. Lond., 25, 1-17. 